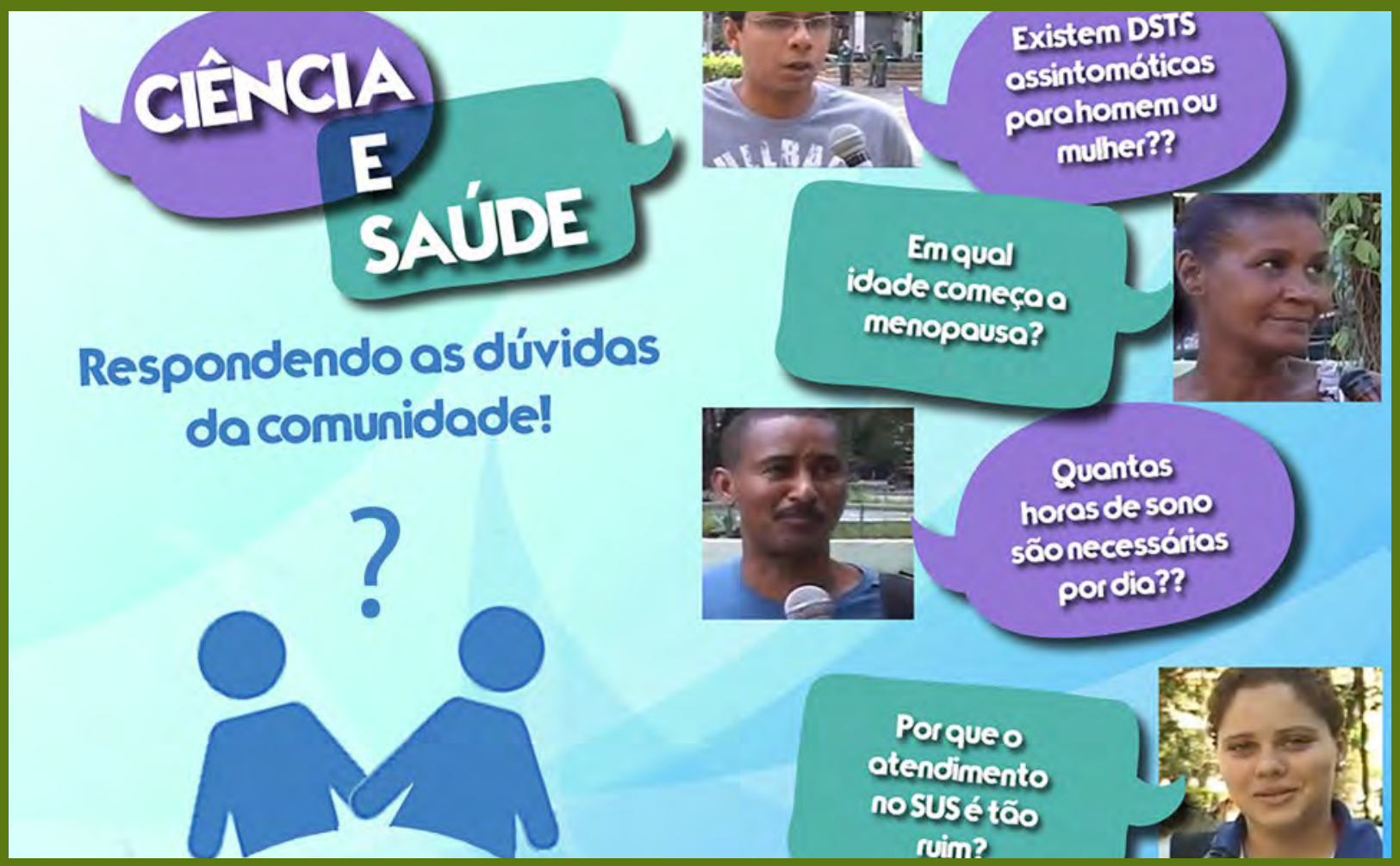

\title{
Espaço Aberto para a Saúde: informação sobre saúde e qualidade de vida para a comunidade
}

Jorge Luiz Lima da Silva - jorgeluizlima@gmail.com¹

João Victor Lima da Silva - limajoao@id.uff.br²

Giulia Lemos de Almeida - giulialemos@id.uff.br ${ }^{3}$

Fernanda Rampe de Oliveira - fernanda_rampe@live.com ${ }^{4}$

\section{RESUMO}

Este relato de experiência apresenta o projeto de extensão "Espaço Aberto para Saúde" (EAS) da Universidade Federal Fluminense (UFF) que, desde 0 ano 2008, contribui para a interação saúde e comunidade. Ele visa promover a educação em saúde como estratégia potencializadora da assistência integral à saúde e trabalha para a produção e divulgação de materiais de ensino, pesquisa e extensão da UFF na área da saúde e cidadania. 0 projeto utiliza mídias sociais para que conteúdos com informações seguras sejam compartilhados entre a população e assim incentive a qualidade de vida. Possui divulgação na mídia por meio do Facebook e YouTube. Durante o ano de 2020, a equipe trabalhou no desenvolvimento de novas propostas como o quadro "Ciência e saúde: pesquisa". Mesmo com os desafios enfrentados em época de pandemia e isolamento social, as gravações estão sendo realizadas de forma remota.

PALAVRAS-CHAVE: saúde pública; qualidade de vida; internet; produção audiovisual; comunicação.

1 Professor da Escola de Enfermagem Aurora de Afonso Costa (EEAAC) - Universidade Federal Fluminense (UFF).

2 Acadêmico de Enfermagem na EEAAC/UFF.

3 Acadêmica de Enfermagem na EEAAC/UFF.

4 Acadêmica de Enfermagem na EEAAC/UFF. 


\title{
An open space for health: information about health and quality of life to the community
}

\begin{abstract}
This experience report presents the extension project "Open Space for Health" (OSH) at the Universidade Federal Fluminense (UFF) that since the2008 contributed the interaction between health and community. It aims at promoting health education as an empowering strategy to integral health assistance as well as cooperating with the promotion and production of teaching, research and extension materials at FFU in the health and citizenship area. The project uses the strategy of social media so the contents with safe information will be shared among the population, and then increase its quality of life. It has been disclosed in the media on Facebook and You Tube. During 2020, the project's responsible staff are developed new ideas such as "Science and health: research". Despite the challenges faced with the challenges faced in times of pandemic and social isolation, the recordings are being made remotely.
\end{abstract}

KEYWORDS: public health; quality of life; internet; audiovisual production; communication.

\section{RELATO DE EXPERIÊNCIA}

A educação em saúde pode ser considerada como estratégia facilitadora para o conhecimento e para o desenvolvimento de habilidades que auxiliem na escolha de hábitos saudáveis, fundamentais para a qualidade de vida. Isso porque os indivíduos são levados à consciência crítica e à avaliação do autocuidado, reconhecendo comportamentos vistos como não benéficos ao seu organismo. A realização de ações educativas em saúde parte da premissa dialógica, criativa e participativa, a qual colabora com a assistência integral (FIGUEIREDO JÚNIOR et al., 2020).

Por definição, a educação em saúde é um conjunto de práticas pedagógicas participativas, que envolvem conhecimentos de diversos campos de atuação e que empodera o sujeito. Nesse contexto, a Política Nacional de Promoção à Saúde foca, entre outros tópicos, na relevância dessa prática ena importância de fortalecer e qualificar a qualidade de vida da família (LIMA et al., 2019).

Com isso, há 12 anos, o professor Jorge Luiz Lima, coordenador desta ação, se uniu a um grupo de três técnicos da TV universitária da Universidade Federal Fluminense (UFF) para verificar possibilidades, mecanismos e estratégias possíveis para que a comunidade da cidade de Niterói e região metropolitana pudesse participar de um programa a ser exibido no canal local. A ideia foi potencializar as ações de orientação em saúde incluindo a população.

Foi criado o projeto de extensão "Espaço Aberto para Saúde" (EAS) da UFF no departamento de "Enfermagem Materno-Infantil e Psiquiatria" e gravado um programa piloto sobre saúde mental em 2/2008, o qual foi veiculado na TV universitária. Nessa primeira etapa, decidiu-se que a educação em saúde seria realizada por intermédio da mídia televisiva. Levou-se em consideração o fato de que a aprendizagem com ferramentas de educação a distância favorece um alcance maior da população, levando conteúdo com qualidade e acessibilidade, com ênfase na comunicação local e comunitária de assuntos que dizem respeito mais diretamente à vida das pessoas, no espaço vivido em seu cotidiano. Sua marca é a proximidade, sintetizada nos sentimentos de pertencimento, de identidades e nos elos do cotidiano. É essa marca que, também, ajuda a garantir a aceitação ou sucesso junto à comunidade (MISKOLCI; PEREIRA, 2019).

Para que haja uma forma de educar qualificada, é relevante discutir essa temática com quem a aplica. Desse modo, abordar a educação em saúde com os profissionais de saúde é de grande valia, visto que a população necessita de cuidado menos técnico, mais humano, com linguagens simples e de fácil compreensão. Ressalte-se, ainda, a necessidade de unir profissionais de diversas áreas para uma orientação interdisciplinar que permita o aprofundamento de discussões e concretização de parcerias entre universidade e comunidade pela via da extensão (SILVA; RIBEIRO, 2019).

A comunidade de Niterói foi consultada nas ruas, nos anos de 2008 e 2009, em áreas do centro da cidade de livre circulação, sobre temas e questões emergentes. Na época, os temas mais comuns eram zoonoses, dengue, infecções sexualmente transmissíveis e atendimento no SUS. Outra forma de interação foi o recebimento de e-mails do público para o projeto e interações pelo Facebook, onde os assuntos eram 
reincidentes. A partir dessa interação, os convidados especialistas da área foram convidados aos estúdios da Unitevê- UFF para gravação de respostas aos questionamentos da comunidade. Os convidados foram professores das universidades federais e estaduais da área metropolitana do Rio de Janeiro (UFF, UFRJ, Uerj, Fiocruz, Unirio). Assim, o projeto se firmou entre a comunidade local desde seu piloto até 2013. Os vídeos eram exibidos via canal web da Unitevê, canal a cabo local e plataforma de vídeo chamada Ufftube, atualmente extinta.

Em 2012, foi criado o Facebook do projeto pelos bolsistas e, posteriormente, o canal do Youtube, em 2013, para que os produtos fossem visualizados sob demanda, o que contribuiu para que a interação saúde e comunidade fosse intensificada. Dessa forma, o projeto contribuiu para que o canal universitário apresentasse programa de TV que abordasse saúde, qualidade de vida e cidadania de forma a divulgar as atividades de docentes, de extensão, de pesquisadores e acadêmicos para comunidade local estadual. Acerca da abrangência de telespectadores, registramos, inclusive, a participação de alunos da universidade que estavam no exterior e que acompanhavam o trabalho realizado. (Figuras 1 e 2)

Figura 1 - Página no Facebook do Espaço aberto para a Saúde, 2000 seguidores, em 04/2021

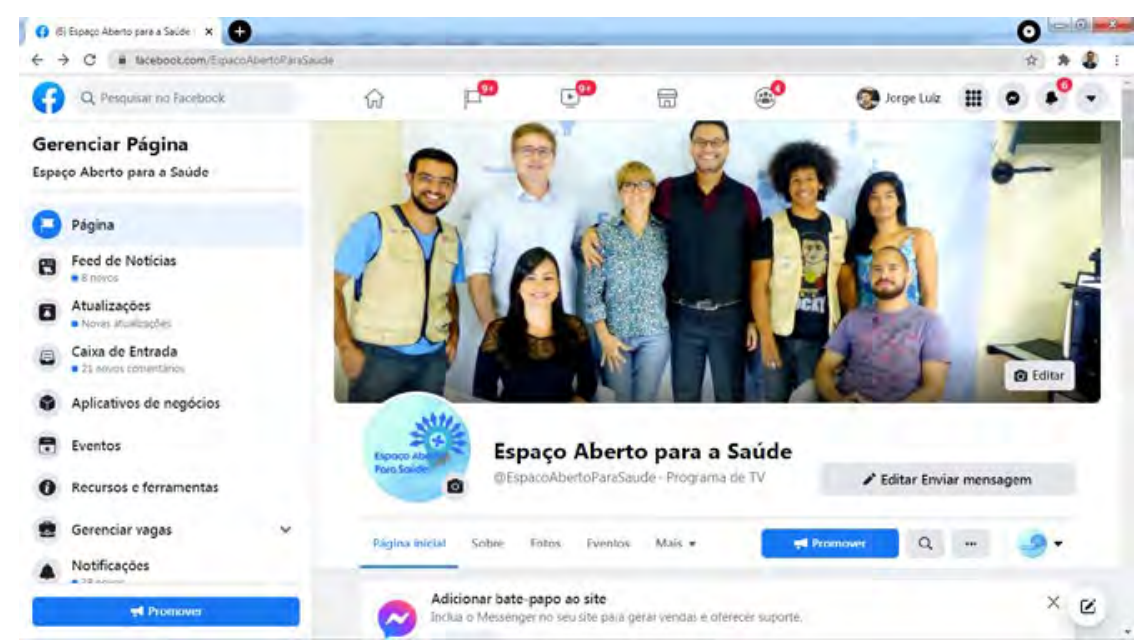

FONTE: https://www.facebook.com/EspacoAbertoParaSaude/.

Figura 2 - Canal no Youtube do "Espaço Aberto para Saúde"

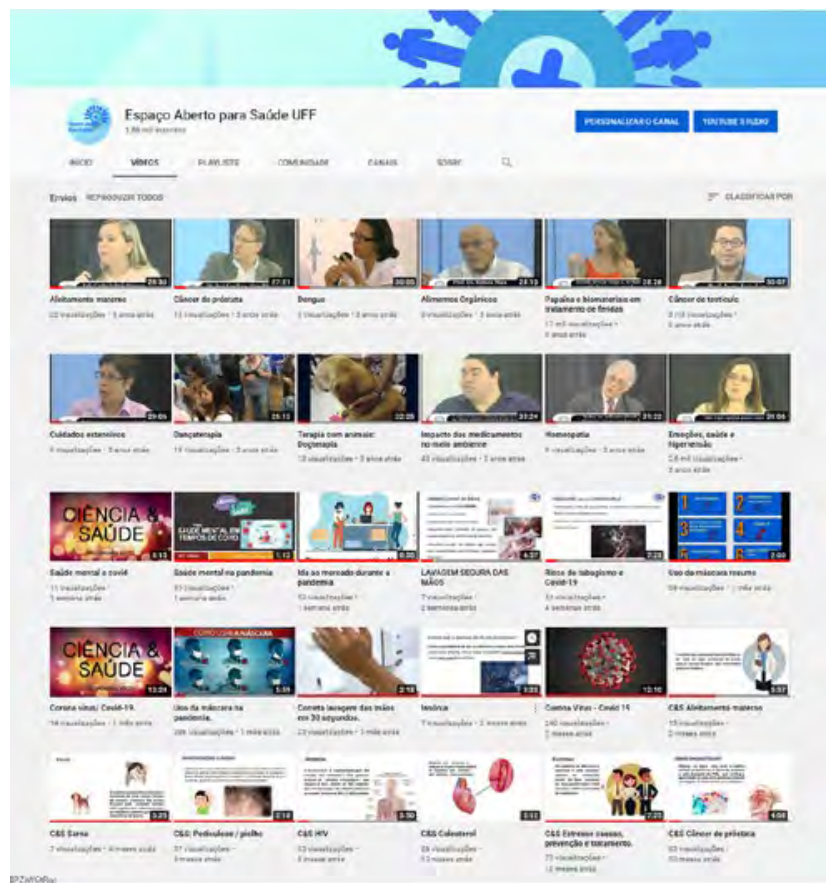

FONTE: https://www.youtube.com/espacoabertoparasaudeuff. 
Em 2013, o projeto de extensão passou a ser "programa" de extensão, devido à união de um conjunto de ações: o próprio EAS, o fornecimento de material à TV universitária (por meio de DVDs), a manutenção da página em mídia social e canal no YouTube; e, por fim, nova ação chamada "Ciência e Saúde" que surgiu para produzir vídeos curtos, legendados, objetivos e animados para serem exibidos nos ambulatórios e salas de espera dos campi da universidade. Neste mesmo ano, as produções passaram a ser exclusivas em parceria com a equipe de comunicação e laboratório de mídia da Pró-reitoria de Extensão e os produtos continuaram sendo enviados a Unitevê para a transmissão. Essa ação ganhou canal próprio no YouTube em 2016, embora as produções sejam replicadas no canal do EAS no Youtube, para atingir maior número de espectadores/ assinantes. (Figura3)

Figura 3 - Canal no Youtube da ação Ciência e Saúde

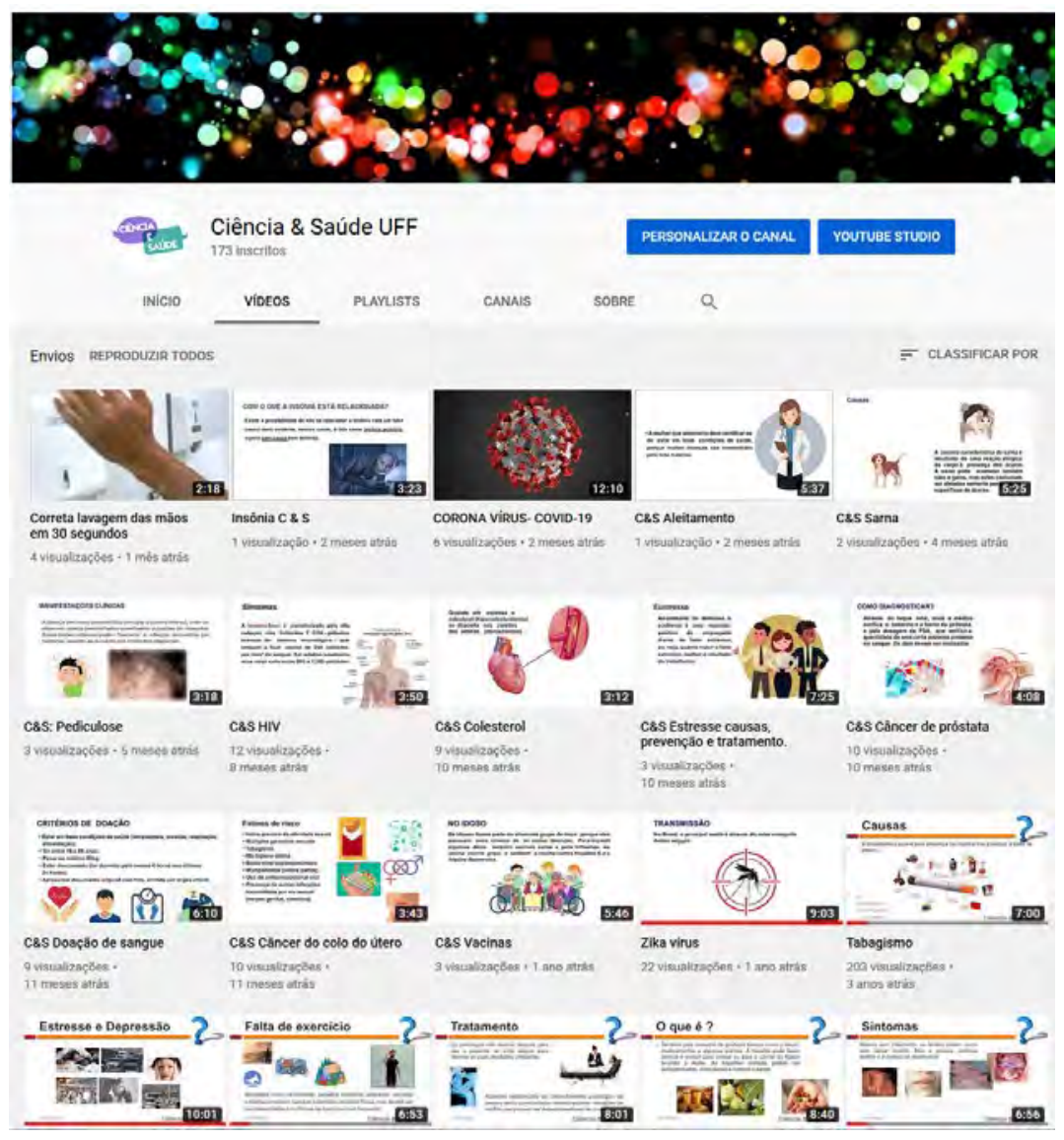

FONTE: https://www.youtube.com/cienciaesaudeuff.

Em 2017, o programa passou a ter uma nova identidade visual criada por um bolsista do curso de graduação em Produção Cultural. As imagens, que ainda são utilizadas atualmente, foram produzidas levando em consideração a opinião dos seguidores do canal. (Figuras 4, 5 e 6)

Figura 4 - Logomarca do Espaço Aberto, 2017.

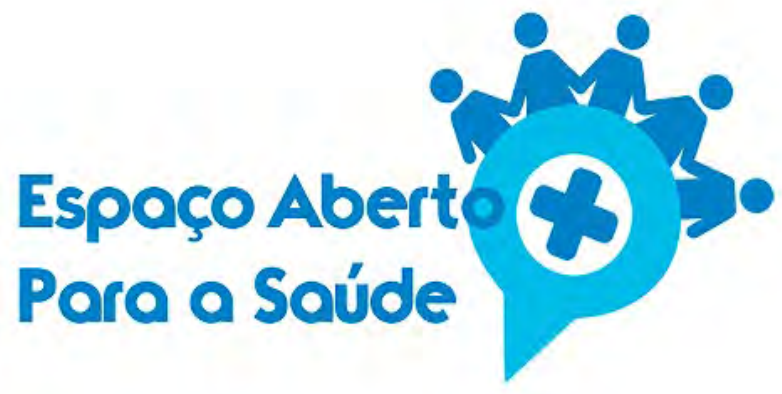


Figura 5 - Logomarca do quadro ciência e saúde, 2017.

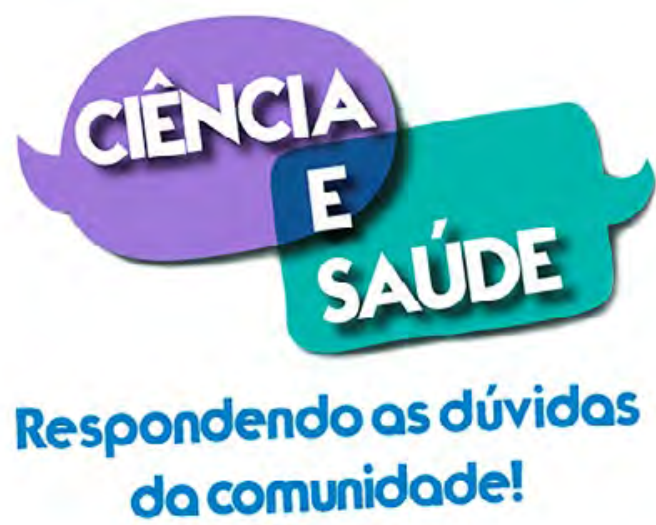

Figura 6 - Plano de Fundo do cenário de gravação, 2017.

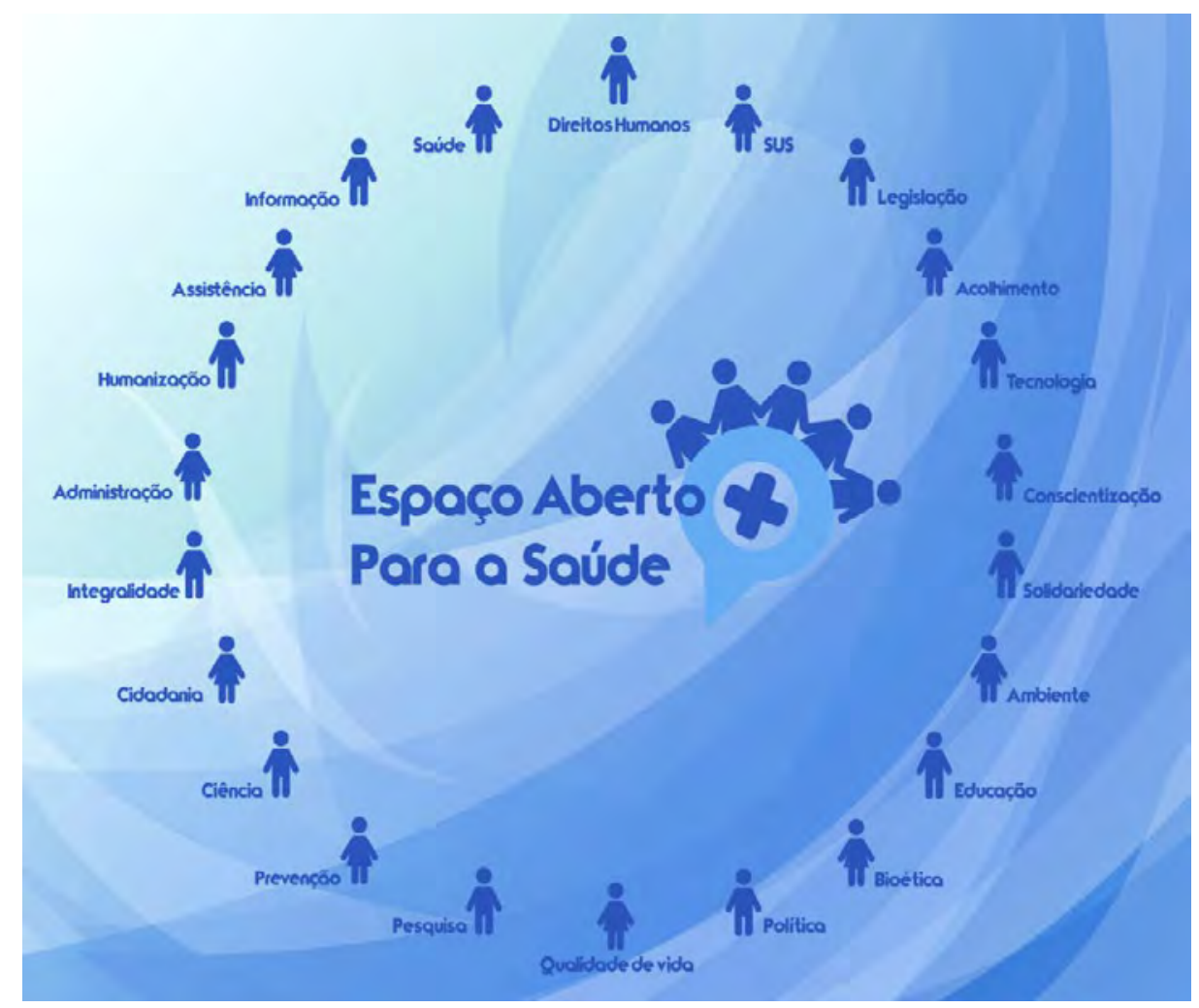

FONTES: Arquivos do programa.

O projeto tem como objetivo geral divulgar a produção de conhecimento de ensino, pesquisa e extensão da UFF na área da saúde e cidadania e possui como objetivos específicos criar e divulgar programas de TV com a temática saúde e qualidade de vida, para serem publicados para a comunidade acadêmica e local.

0 EAS favorece que a universidade ocupe mais espaço na mídia. Com isso, cria-se a possibilidade de levar informações baseadas em critérios científicos para a comunidade e de mostrar sua presença na mídia, marcando posição no campo de excelência com a participação da comunidade acadêmica. OEAS éformado por profissionais de saúde, professores universitários do curso de Enfermagem e Medicina, técnicos, servidores e alunos de graduação em Produção cultural, Comunicação social, Enfermagem e, o mais importante, por moradores, seguidores do canal e pela comunidade.

A dinâmica funciona da seguinte forma: levantamento do tema de interesse por demanda da comunidade da região metropolitana de Niterói, escuta ativa, gravações, recebimento de e-mails, contatos 
pelas redes sociais, confecção do roteiro por acadêmicos de Enfermagem, convite do profissional a ser entrevistado, agendamento da filmagem em estúdio, gravação - com apresentação do coordenador e auxílio de acadêmicos de Cinema, Produção cultural, Jornalismo e Enfermagem, edição pelos bolsistas de Cinema e Produção Cultural, revisão do material pelo coordenador e convidados, publicação do vídeo no canal e compartilhamento. 0 material é disponibilizado quinzenalmente entre os meses de maio a dezembro. As dúvidas e interações ocorrem ininterruptamente, assim como sugestão de novos temas por e-mails, chat do Facebook e comentários no YouTube. Todos os participantes entrevistados assinam termo de cessão de imagem. (Figura 7).

Figura 7 - Participação da comunidade em praça pública, 2015.

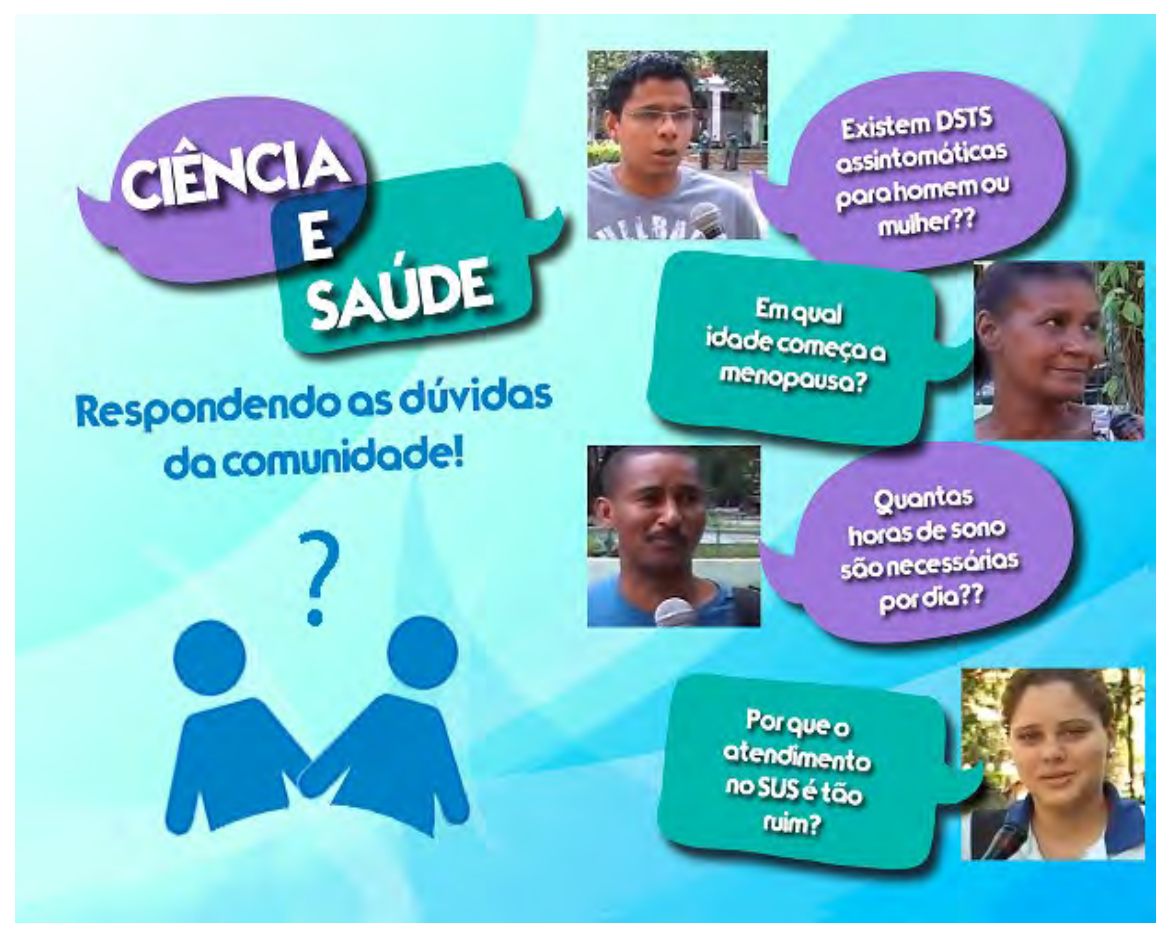

FONTE: Arquivo digital do projeto.

0 canal do EAS conta com mais de 100 vídeos e cerca de 1.900 de inscritos. 0 total de visualizações do canal é de aproximadamente (a soma das visualizações de todos os vídeos da página)120 mil. 0 vídeo com tema "colostomia (cuidados e tipos de ostomias)" foi o mais visualizado, assistido 72 mil vezes - dados de junho de 2020.

As últimas gravações contaram com pesquisadores e especialistas respondendo questões sobre zoonoses, segurança do paciente, pessoas em situações de rua, aleitamento materno, cuidadores de idosos, SUS e financiamento em tempos de crise, cuidados com colostomia, toxicologia, vacinação de adultos, acompanhamento de parto por doulas, dentre outros.

Coma criação da ação extensionista “Ciência e Saúde UFF”, o material educativo ganhou apresentações animadas sobre os assuntos discutidos anteriormente com especialistas para que a população recebesse informações variadas de forma ilustrada, dinâmica, pontual e resumida, como dengue, ansiedade e transplante de órgãos. Outros exemplos estão expostos no quadro 1 e quadro 2. 
Quadro 1 - Vídeos do canal Espaço Aberto para Saúde, 2020.

\begin{tabular}{|c|c|c|}
\hline $\begin{array}{c}\text { VIDEO } \\
\text { DATA DE PUBLICACCÃO } \\
\text { NÜMERO DE ACESSOS }\end{array}$ & $\begin{array}{l}\text { PROFISSIONAL } \\
\text { CONVIDADO }\end{array}$ & LINK DE ACESSO \\
\hline $\begin{array}{l}\text { Isolamento social e pandemia; } \\
30 \text { out. } 2020 ; \\
392 \text { visualizações. }\end{array}$ & $\begin{array}{l}\text { Profa. Dra. Rose Mary } \\
\text { Costa Rosa. }\end{array}$ & $\begin{array}{l}\text { Disponível em: https://youtu.be/ } \\
\text { z4FdNxDOPYO Acesso em: } 21 \text { maio de } 2021 .\end{array}$ \\
\hline $\begin{array}{l}\text { Parto e puerpério na pandemia; } \\
16 \text { out. } 2020 ; \\
70 \text { visualizações. }\end{array}$ & $\begin{array}{l}\text { Enfermeira obstétrica } \\
\text { Patrícia Salles. }\end{array}$ & $\begin{array}{c}\text { Disponível em: https://youtu.be/ } \\
\text { ndMXGJTa4cM Acesso em: } 21 \text { maio de } 2021 .\end{array}$ \\
\hline $\begin{array}{l}\text { Banco de leite humano e a } \\
\text { pandemia; } \\
09 \text { out. 2020; } \\
31 \text { visualizações. }\end{array}$ & $\begin{array}{l}\text { Enfa. Maria Bertila } \\
\quad \text { Lutterbach. }\end{array}$ & $\begin{array}{l}\text { Disponível em: https://youtu.be/FXQLauZ Cjk } \\
\text { Acesso em: } 21 \text { maio de } 2021 .\end{array}$ \\
\hline $\begin{array}{l}\text { Urgência e emergência na pandemia; } \\
02 \text { out. } 2020 ; \\
40 \text { visualizações. }\end{array}$ & $\begin{array}{l}\text { Enf. Fabiano Mizael } \\
\text { Carolindo. }\end{array}$ & $\begin{array}{l}\text { Disponivel em: https://youtu.bel } \\
\text { MY6xqPIB4FU Acesso em: } 21 \text { maio de } 2021 .\end{array}$ \\
\hline $\begin{array}{l}\text { Atendimento psicológico ao } \\
\text { trabalhador da saúde; } \\
18 \text { set. } 2020 ; \\
96 \text { visualizações. }\end{array}$ & $\begin{array}{l}\text { Psicóloga Irene } \\
\text { Trajtenberg. }\end{array}$ & $\begin{array}{l}\text { Disponível em: } \text { https://youtu.be/ } \\
\text { oJfgFHMpY8g Acesso em: } 21 \text { maio de } 2021 .\end{array}$ \\
\hline
\end{tabular}

Violência contra à mulher;

11 set. 2020;

51 visualizações.

Dr. Leônidas

Albuquerque.
Disponível em: https://youtu.be/DMBFt9rjZaE Acesso em: 21 maio de 2021.
Doação de órgãos e pandemia;

04 set. 2020 ;

66 visualizações.
Enf. Fabrício Oliveira. Disponível em: https://youtu.bel IPEWJGcUdLQ Acesso em: 21 maio de 2021.

Coronavírus e cuidados na pediatria;

Enfa. e Capitã Tenente da Marinha Vanessa Galvão.
Disponível em: https://youtu.be/5UCWXWUOgg Acesso em: 21 maio de 2021.
Disponível em: https://youtu.be/ScNVId7UWM

Acesso em: 21 maio de 2021.
Saúde do índio na pandemia 2020; 16 ago. 2020;

233 visualizações.
Dr. Andrey Moreira Cardoso.
Saúde da Mulher na pandemia; 07 ago. 2020;

147 visualizações.
Prof. Dr. Valdcyr Herdy Disponível em: https://youtu.be/Gw S2SrO M Alves.
Acesso em: 21 maio de 2021. 
Quadro 2 - Vídeos do canal Espaço Aberto para Saúde - Ciência \& Saúde, 2020.

\begin{tabular}{|c|c|}
\hline $\begin{array}{c}\text { VIDDEO } \\
\text { NÜMEROS DE ACESSOS }\end{array}$ & $\begin{array}{l}\text { DATA DE PUBLICAÇÃO } \\
\text { LINK DE ACESSO }\end{array}$ \\
\hline $\begin{array}{l}\text { Ciência \& Saúde Pesquisa: Marcela Chagas PSF; } \\
82 \text { visualizações. }\end{array}$ & $\begin{array}{c}23 \text { out. 2020; } \\
\text { Disponível em: https://youtu.be/3ulLv6_P104 } \\
\text { Acesso em: } 21 \text { maio de } 2021 .\end{array}$ \\
\hline $\begin{array}{l}\text { Cuidado com alimentos na pandemia; } \\
733 \text { visualizações. }\end{array}$ & $\begin{array}{c}11 \text { jun. } 2020 ; \\
\text { Disponível em: https://youtu.be/Chl8IGBce_s } \\
\text { Acesso em: } 21 \text { maio de } 2021 .\end{array}$ \\
\hline $\begin{array}{l}\text { Saúde mental e covid; } \\
106 \text { visualizações. }\end{array}$ & $\begin{array}{c}23 \text { mai. } 2020 ; \\
\text { Disponível em: https://youtu.be/-QD154eV8WU } \\
\text { Acesso em: } 21 \text { maio de } 2021 .\end{array}$ \\
\hline $\begin{array}{l}\text { Saúde mental na pandemia; } \\
2.888 \text { visualizações. }\end{array}$ & $\begin{array}{c}22 \text { mai.2020; } \\
\text { Disponível em: https://youtu.be/qRV2eH7PwaA } \\
\text { Acesso em: } 21 \text { maio de } 2021 .\end{array}$ \\
\hline $\begin{array}{l}\text { Ida ao mercado durante a pandemia; } \\
69 \text { visualizações. }\end{array}$ & $\begin{array}{c}22 \text { mai. } 2020 ; \\
\text { Disponível em: https://youtu.be/uzDOShGvNiA } \\
\text { Acesso em: } 21 \text { maio de } 2021 .\end{array}$ \\
\hline $\begin{array}{l}\text { Lavagem segura das mãos; } \\
18 \text { visualizações. }\end{array}$ & $\begin{array}{c}10 \text { mai. } 2020 ; \\
\text { Disponível em: } \underline{\text { https://youtu.be/7El-4Ee-hU4 }} \\
\text { Acesso em: } 21 \text { maio de } 2021 .\end{array}$ \\
\hline $\begin{array}{l}\text { Risco do tabagismo e Covid-19; } \\
806 \text { visualizações. }\end{array}$ & 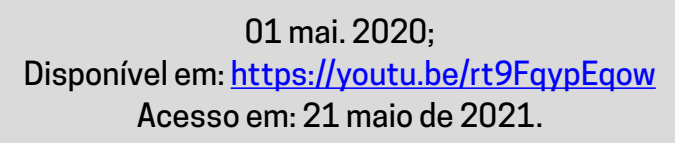 \\
\hline $\begin{array}{l}\text { Uso da máscara na pandemia; } \\
1.902 \text { visualizações. }\end{array}$ & $\begin{array}{l}25 \text { abr. 2020; } \\
\text { Disponível em: https://youtu.be/SnmMLI r4k8 } \\
\text { Acesso em: } 21 \text { maio de } 2021 .\end{array}$ \\
\hline $\begin{array}{l}\text { Coronavírus/Covid-19; } \\
57 \text { visualizações. }\end{array}$ & $\begin{array}{c}29 \text { abr. 2020; } \\
\text { Disponível em: } \underline{\text { https://youtu.be/VPtjcwa8vgk }} \\
\text { Acesso em: } 21 \text { maio de } 2021 .\end{array}$ \\
\hline $\begin{array}{l}\text { Coronavírus - Covid 19; } \\
591 \text { visualizações. }\end{array}$ & $\begin{array}{c}20 \text { mar. } 2020 ; \\
\text { Disponível em: https://youtu.be/HOuQyuh4Lbl } \\
\text { Acesso em: } 21 \text { maio de } 2021 .\end{array}$ \\
\hline
\end{tabular}

FONTE: 0 s autores.

No ano de 2019, foram abordados temas como febre mayaro, insônia, obesidade, práticas resistentes e infarto. 0 canal conta também com vídeos que abordam temáticas como HIV, colesterol, estresse, câncer de próstata, doação de sangue, câncer de colo do útero, zika, vacinas e covid-19. Vale lembrar que os temas são escolhidos a partir das demandas da população, dos assuntos de interesse e indicações dos inscritos no canal.

Tais produções são legendadas e reproduzidas nas TVs instaladas pelos campi da universidade, nas salas da reitoria, no hospital universitário, nos ambulatórios e refeitórios. Em 2020, o "Ciência e Saúde UFF", vendo a necessidade de abordar temas voltados para a covid-19, devido à pandemia, abordou temas como saúde mental, higienização das mãos, uso correto de máscaras, dentre outros.

A iniciativa EAS busca desenvolver temas voltados ao atendimento das demandas da população e compreender a relação entre a história de vida e o processo dinâmico das condições concretas das pessoas. 
Em 2018, o projeto recebeu o prêmio "Josué de Castro 5 de Extensão" (2ํ lugar) na área de comunicação, na Semana Acadêmica da UFF, demonstrando sua relevância e visibilidade dentro do âmbito acadêmico.

0 novo desafio agora é divulgar, no "Ciência e Saúde", um novo quadro chamado "Ciência e Saúde: pesquisa" que visa divulgar para a população os principais resultados de pesquisas e estudos do mestrado em "Saúde coletiva" da UFF. 0 piloto foi gravado no ano de 2020 e está sendo editado.

Faz-se necessário utilizar estratégias a fim de identificar lacunas que, por meio da educação, possam ser preenchidas. 0 projeto favorece o aprendizado dos seus contribuintes, porque além de abordar questões referentes à saúde e estar relacionado à divulgação à comunidade, permite a ampliação e a troca de saberes com diversas áreas de conhecimento. Dessa forma, a interdisciplinaridade se mostra presente em cada etapa deste programa extensionista.

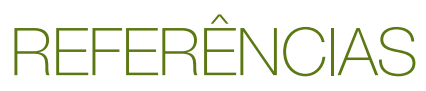

FIGUEIREDO JÚNIOR, Adilson Mendes, et al. Percepção de acadêmicos de Enfermagem sobre educação em saúde na perspectiva da qualificação do cuidado. Revista Eletrônica Acervo Saúde [Internet], v. 12, n. 1, p. e1964, 2020. Disponível em: <https://acervomais.com.br/index.php/saude/article/view/1964>.

LIMA, Geisa Carla de Brito Bezerra, et al. Educação em saúde e dispositivos metodológicos aplicados na assistência ao Diabetes Mellitus. Saúde em Debate [Internet], v. 43, n. 120, p. 150-158, 2019. Disponível em: <http://www.scielo.br/scielo.php?script=sci_arttext\&pid=S0103-11042019000100150\&lng=en.>

MISKOLCI, Richard; PEREIRA, Pedro Paulo Gomes. Educação e Saúde em disputa: movimentos anti-igualitários e políticas públicas. Interface - Comunicação, Saúde, Educação [Internet], v. 23, p. 1-14, 2019. Disponível em: < http://www.scielo.br/scielo.php?script=sci_arttext\&pid=S1414-32832019000100400\&lng=en.>.

SILVA, Gabriela Aparecida; RIBEIRO, Isabely Karoline da Silva; TEIXEIRA, Graziela Silveira. Educação em saúde em um encontro de gestantes: relato de experiência de acadêmicas de Enfermagem. Caminho Aberto: revista de extensão do IFSC [Internet], e. 06, n. 11, p. 57-61, 2019. Disponível em: <https://periodicos.ifsc.edu.br/index. php/caminhoaberto/article/view/2624>.

5 Prêmio Josué de Castro de Extensão é entregue aos artigos que obtiveram as melhores avaliações entre os trabalhos apresentados na Semana de Extensão da UFF (Semext). 